\title{
Analysis of gene expression profile microarray data in complex regional pain syndrome
}

\author{
WULIN TAN $^{1 *}$, YIYAN SONG $^{2 *}$, CHENGQIANG MO $^{3}$, SHUANGJIAN JIANG $^{3}$ and ZHONGXING WANG ${ }^{1}$ \\ ${ }^{1}$ Department of Anesthesiology, The First Affiliated Hospital of Sun Yat-sen University; ${ }^{2}$ Zhongshan \\ School of Medicine, Sun Yat-sen University; ${ }^{3}$ Department of Urology, The First Affiliated \\ Hospital of Sun Yat-sen University, Guangzhou, Guangdong 510080, P.R. China
}

Received April 6, 2016; Accepted March 16, 2017

DOI: $10.3892 / \mathrm{mmr} .2017 .6950$

\begin{abstract}
The aim of the present study was to predict key genes and proteins associated with complex regional pain syndrome (CRPS) using bioinformatics analysis. The gene expression profiling microarray data, GSE47603, which included peripheral blood samples from 4 patients with CRPS and 5 healthy controls, was obtained from the Gene Expression Omnibus (GEO) database. The differentially expressed genes (DEGs) in CRPS patients compared with healthy controls were identified using the GEO2R online tool. Functional enrichment analysis was then performed using The Database for Annotation Visualization and Integrated Discovery online tool. Protein-protein interaction (PPI) network analysis was subsequently performed using Search Tool for the Retrieval of Interaction Genes database and analyzed with Cytoscape software. A total of 257 DEGs were identified, including 243 upregulated genes and 14 downregulated ones. Genes in the human leukocyte antigen (HLA) family were most significantly differentially expressed. Enrichment analysis demonstrated that signaling pathways, including immune response, cell motion, adhesion and angiogenesis were associated with CRPS. PPI network analysis revealed that key genes, including early region $1 \mathrm{~A}$ binding protein p300 (EP300), CREB-binding protein (CREBBP), signal transducer and activator of transcription (STAT)3, STAT5A and integrin $\alpha \mathrm{M}$ were associated with CRPS. The results suggest that the immune response may therefore serve an important role in CRPS development. In addition, genes in the HLA family, such as HLA-DQB1 and HLA-DRB1,
\end{abstract}

Correspondence to: Dr Zhongxing Wang, Department of Anesthesiology, The First Affiliated Hospital of Sun Yat-sen University, 58 Zhongshan Road 2, Guangzhou, Guangdong 510080, P.R. China

E-mail: doctorwzx@126.com

*Contributed equally

Key words: complex regional pain syndrome, differentially expressed genes, functional enrichment, protein-protein interaction network, module identification may present potential biomarkers for the diagnosis of CRPS. Furthermore, EP300, its paralog CREBBP, and the STAT family genes, STAT3 and STAT5 may be important in the development of CRPS.

\section{Introduction}

Complex regional pain syndrome (CRPS) is an uncommon chronic pain condition that develops following trauma. The features include limb pain, allodynia, hypersensitivity, motor abnormality and trophic alterations (1). Women are affected more commonly than men, and postmenopausal women demonstrate the highest risk (2). CRPS is classified into two subtypes depending on the presence of peripheral nerve injury. CRPS type I without peripheral nerve injury is more common than type II, which presents as peripheral nerve injury (3). In addition, CRPS is categorized into 'warm' or 'cold' CRPS depending on whether skin temperature is increased or decreased (4). Although CRPS is designated as an orphan disease in the general population, it affects $\sim 4-7 \%$ patients experiencing limb fractures (5). CRPS is a burden on the healthcare system and society. A number of different therapeutic strategies have been applied to treat CRPS, including pharmacological, interventional and psychological methods; however, there evidence to support their efficiency of these methods is insufficient (5). The pathogenesis of CRPS is unclear (6), however several mechanisms have are thought to be involved, including inflammation, neurogenic inflammation and alterations of the central nerve system (6). Goebel et al (7) elucidated the association between inflammation, immune response and CRPS, and an autoimmune model was proposed. Oaklander et al (8) proposed that CRPS is associated with distal degeneration of small diameter peripheral axons. Barad et al (9) demonstrated that brain structure was involved in CRPS. However, little is known about how these mechanisms interact to lead to CRPS. Therefore, identifying key genes or signaling pathways involved in the development of CRPS may facilitate elucidation of the integrated mechanism of CRPS and enable the development of targeted therapies.

Genome-wide expression profiling of CPRS was recently performed by Jin et al (10). The authors collected and analyzed peripheral blood examples from healthy controls and patients with CRPS. Differentially expressed genes (DEGs) 
were identified using microarray analysis, and out of those identified, 6 genes were selected for further confirmation by reverse transcription-quantitative polymerase chain reaction analysis. The authors focused on a significantly upregulated DEG (matrix metallopeptidase 9), thought to be associated with pain progression in CRPS. However, the gene/protein interaction networks involved in CRPS remain unknown, which is necessary to elucidate how inflammation, neurogenic inflammation and alterations of the central nervous system are involved in CRPS. Therefore, the authors of the present study constructed a protein-protein interaction network in order to identify the specific molecular interactions involved in CRPS.

In the present study, the GSE47603 gene expression profiling microarray data deposited by Jin et al (10) was obtained and used to identify CRPS-associated genes in patients with CRPS. The microarray data was used to identify key DEGs associated with CRPS by employing comprehensive analysis methods in order to enrich the functions and signaling pathways of identified DEGs. In addition, a protein-protein interaction (PPI) network was constructed and analyzed to identify hub genes. The aim of the current study was to identify several key genes associated with the disease, and examine their potential function in the development of CRPS by expression profile analysis. The results may facilitate the identification of potential targets for the diagnosis and treatment of CRPS.

\section{Materials and methods}

Microarray data. The GSE47603 microarray data, deposited by Jin et al (10) was obtained from the Gene Expression Omnibus (GEO; http://www.ncbi.nlm.nih.gov/geo). This microarray used the GPL10558 platform (Illumina HumanHT-12 v4 expression beadchip; Illumina, Inc., San Diego, CA, USA). A total of 9 peripheral blood samples from 4 patients with CRPS and 5 healthy controls were included in the genome-wide expression profiling array, as described previously (10). Out of the patients with CRPS, 2 patients presented with CRPS type I and 2 patients presented with CRPS type II. Patients who received medication of CRPS were included; however, those with additional neurogenic disorders caused by this medication were excluded. The control subjects did not present with infectious disease, pain disorders and had not undergone recent surgery.

Data processing. Array data export, processing and analysis was performed using the Gene Expression Module (version, 1.9.0) of Illumina GenomeStudio software (version, 2011.1; Illumina, Inc.). Data were already quantile-normalized. Significant DEGs were identified by comparing profile data from patients with CRPS with that of control subjects using the online analysis tool, GEO2R (http://www.ncbi.nlm.nih. gov/geo/geo2r/, version R 3.2.3, Biobase 2.30.0, GEOquery 2.40.0, limma 3.26.8), as was accessible from the GEO website. This tool uses the linear models for microarray analysis package to identify DEGs and provides t-statistics and P-values. Benjamini and Hochberg's false discovery rate was applied to adjust the P-values. DEGs with adjusted P-values of $<0.05$ and fold change (FC) values of $>1.5$ were considered to be significant.
Gene ontology (GO) enrichment and Kyoto encyclopedia of genes and genomes (KEGG) pathway analysis. The GO database (http://geneontology.org) is a large-scale collection of genomic data divided into 3 categories according to biological process (BP), molecular function and cellular component. KEGG (http://www.genome/ad.jp/kegg/) is a pathway-associated database for gene classification. The Database for Annotation Visualization and Integrated Discovery (DAVID, https://david.ncifcrf.gov/) integrates biological data and analysis tools to provide systematic functional annotation for a large number of genes and proteins.

To analyze the putative functions of identified DEGs, GO annotation associated with BP and KEGG pathway enrichment analyses were performed using the online DAVID tool version $6.7(11,12)$. A P-value of $<0.05$ and gene counts of $>2$ were considered to be significant.

PPI construction. The Search Tool for the Retrieval of Interaction Genes (STRING; http://string.embl.de/) database collects comprehensive information regarding predicted and experimental interactions between proteins in a given cell. In the present study, DEGs were mapped in the STRING database version, 10.0 (13) to construct a PPI network, which provides an improved understanding of the functional organization of the proteome. A combined score of $>4$ was set as the threshold. The PPI network was subsequently analyzed using Cytoscape (http://www.cytoscape.org/, version, 3.3.0) software. The degree of connectivity of each node in the PPI network was calculated, and the hub nodes were identified. Genes were clustered using the MCODE Cytoscape plugin (version, 1.4.1).

\section{Results}

DEG analysis. Using adjusted $\mathrm{P}$-values of $<0.05$ and FC values of $>1.5$, a total of 257 DEGs were identified in blood samples from patients with CRPS when compared with the controls. This included 243 upregulated and 14 downregulated genes (Tables I and II). The most significantly upregulated and downregulated genes were human leukocyte antigen (HLA)-DRB1 and HLA-DQB1, respectively, which belong to the HLA family. An additional member of the HLA family, HLA-DRB4, was significantly downregulated in CRPS samples when compared with controls (Table II).

Functional enrichment analysis. The over-represented GO-BP terms of DEGs were primarily associated with antigen processing and presentation, the immune response process, the integrin-mediated signaling pathway, cell motion and adhesion, angiogenesis, and cell-substrate junction assembly (Table III). In addition, DEGs were enriched in KEGG pathways including viral myocarditis, systemic lupus erythematosus, asthma, allograft rejection, graft-vs.-host disease, type I diabetes mellitus, intestinal immune network for immunoglobulin A production, autoimmune thyroid disease, antigen processing and presentation and hematopoietic cell lineage (Table IV).

PPI analysis. The PPI network generated using the STRING database included 155 nodes and 400 edges (Fig. 1). Topological 
Table I. Significantly upregulated differentially expressed genes $(n=15)$.

\begin{tabular}{lccc}
\hline Illumina ID & Adjusted P-value & $\operatorname{logFC}$ & Gene symbol \\
\hline ILMN_1715169 & 0.0000060 & 3.8971524 & HLA-DRB1 \\
ILMN_1738075 & 0.0026403 & 1.3041826 & CMIP \\
ILMN_1670130 & 0.0015978 & 1.2499989 & ARID3A \\
ILMN_1722872 & 0.0033398 & 1.1720856 & MYH9 \\
ILMN_2371169 & 0.0026403 & 1.1696512 & ZYX \\
ILMN_1696643 & 0.0026403 & 1.0804514 & TLN1 \\
ILMN_1760027 & 0.0026403 & 0.9688758 & WAS \\
ILMN_1811823 & 0.0026403 & 0.9556934 & MED25 \\
ILMN_1663618 & 0.0026403 & 0.9497475 & STAT3 \\
ILMN_1800425 & 0.0026403 & 0.9133604 & SLC9A1 \\
ILMN_1777906 & 0.0030662 & 0.9120812 & MAP7D1 \\
ILMN_1725534 & 0.0026403 & 0.8451986 & ACTN4 \\
ILMN_1682930 & 0.0026403 & 0.8188014 & SIPA1 \\
ILMN_2275098 & 0.0026403 & 0.7684677 & DTX2 \\
ILMN_1764788 & 0.0026403 & 0.7212105 & TNFRSF1B
\end{tabular}

Benjamini and Hochberg's false discovery rate was applied to adjust the P-value. FC, fold-change.

Table II. Significantly downregulated differentially expressed genes $(n=14)$.

\begin{tabular}{lccc}
\hline Illumina ID & Adjusted P-value & $\operatorname{logFC}$ & Gene symbol \\
\hline ILMN_1661266 & 0.0151182 & -2.4675689 & HLA-DQB1 \\
ILMN_2356991 & 0.0196116 & -1.2897218 & CD47 \\
ILMN_1718766 & 0.0086679 & -0.8003821 & MT1F \\
ILMN_3241953 & 0.0341073 & -0.7595469 & GGACT \\
ILMN_1741133 & 0.0161371 & -0.7282374 & NME1 \\
ILMN_1780368 & 0.0251894 & -0.7091328 & GPR18 \\
ILMN_1676575 & 0.0251784 & -0.6922376 & IKZF1 \\
ILMN_1712298 & 0.0169227 & -0.6912831 & ANKRD46 \\
ILMN_1726460 & 0.0136377 & -0.6765224 & RPL14 \\
ILMN_1715661 & 0.0211196 & -0.6750304 & TFAM \\
ILMN_1771333 & 0.0121527 & -0.6515932 & CD47 \\
ILMN_1752592 & 0.0493698 & -0.6494842 & HLA-DRB4 \\
ILMN_2326273 & 0.0241474 & -0.6120625 & CHI3L2 \\
ILMN_1653026 & 0.0196116 & -0.6098733 & PLAC8 \\
\hline
\end{tabular}

Benjamini and Hochberg's false discovery rate was applied to adjust the P-value. FC, fold-change.

structure analysis performed by Cytoscape software revealed the degree of genes in the PPI network. Nodes with a higher degree are more connected with other nodes, and they are considered to contribute to the stability of the network and are designated as hub nodes. In the present study, genes with a higher degree included adenovirus early region 1A binding protein p300 (EP300), CREB-binding protein (CREBBP), signal transducer and activator of transcription (STAT) 3 and STAT5A of the STAT protein family, interleukin 8 (IL8) and integrin $\alpha \mathrm{M}$ (ITGAM).
A total of 3 modules with a score of $>4$ were identified using the MCODE plugin (Fig. 2). Cluster 1 exhibited the highest score, and DEGs, such as EP300, CREBBP, STAT3, STAT5A and ITGAM were included. Enrichment analysis demonstrated that the GO-BP terms enriched by these genes were associated with regulation of transcription, biosynthesis, hormone stimuli responses and cell differentiation (Table V). DEGs in cluster 2 primarily included ITGAM, retinoic acid receptor $\alpha$, integrin subunit $\alpha 2 \mathrm{~B}$ and DNA-directed RNA polymerase II subunit RPB1 (Fig. 2), which were enriched 
Table III. Most significant GO-biological process terms enriched by differentially expressed genes $(n=15)$.

\begin{tabular}{|c|c|c|c|}
\hline GO ID & Biological process & Count & P-value \\
\hline GO:0007229 & Integrin-mediated signaling pathway & 9 & $5.01 \times 10^{-6}$ \\
\hline GO:0007010 & Cytoskeleton organization & 20 & $9.05 \times 10^{-6}$ \\
\hline GO:0030036 & Actin cytoskeleton organization & 14 & $1.45 \times 10^{-5}$ \\
\hline GO:0030029 & Actin filament-based process & 14 & $2.88 \times 10^{-5}$ \\
\hline GO:0006955 & Immune response & 24 & $7.43 \times 10^{-5}$ \\
\hline GO:0006952 & Defense response & 22 & $1.12 \times 10^{-4}$ \\
\hline GO:0022604 & Regulation of cell morphogenesis & 9 & $4.51 \times 10^{-4}$ \\
\hline GO:0007242 & Intracellular signaling cascade & 33 & $4.52 \times 10^{-4}$ \\
\hline GO:0045321 & Leukocyte activation & 12 & $5.23 \times 10^{-4}$ \\
\hline GO:0045596 & Negative regulation of cell differentiation & 11 & $8.13 \times 10^{-4}$ \\
\hline GO:0008360 & Regulation of cell shape & 6 & $8.53 \times 10^{-4}$ \\
\hline GO:0001775 & Cell activation & 12 & $2.06 \times 10^{-3}$ \\
\hline GO:0006928 & Cell motion & 16 & $2.36 \times 10^{-3}$ \\
\hline GO:0035023 & Regulation of Rho protein signal transduction & 7 & $2.42 \times 10^{-3}$ \\
\hline GO:0051056 & Regulation of small GTPase mediated signal transduction & 11 & $2.56 \times 10^{-3}$ \\
\hline
\end{tabular}

GO, Gene Ontology; GTP, guanosine triphosphate.

Table IV. Most significant KEGG terms enriched by differentially expressed genes $(n=15)$.

\begin{tabular}{llrr}
\hline KEGG ID & \multicolumn{1}{c}{ KEGG term } & Count & P-value \\
\hline hsa04810 & Regulation of actin cytoskeleton & 14 & $9.87 \times 10^{-5}$ \\
hsa04670 & Leukocyte transendothelial migration & 9 & $1.13 \times 10^{-3}$ \\
hsa04666 & Fc- R-mediated phagocytosis & 8 & $1.43 \times 10^{-3}$ \\
hsa04520 & Adherens junction & 7 & $2.38 \times 10^{-3}$ \\
hsa05221 & Acute myeloid leukemia & 6 & $3.57 \times 10^{-3}$ \\
hsa05416 & Viral myocarditis & 6 & $8.45 \times 10^{-3}$ \\
hsa04330 & Notch signaling pathway & 5 & $9.52 \times 10^{-3}$ \\
hsa05200 & Pathways in cancer & 13 & $1.32 \times 10^{-2}$ \\
hsa04142 & Lysosome & 7 & 6 \\
hsa04640 & Hematopoietic cell lineage & 8 & $1.78 \times 10^{-2}$ \\
hsa04630 & Jak-STAT signaling pathway & 9 & $2.03 \times 10^{-2}$ \\
hsa04510 & Focal adhesion & 5 & $2.66 \times 10^{-2}$ \\
hsa05211 & Renal cell carcinoma & 8 & $3.59 \times 10^{-2}$ \\
hsa04144 & Endocytosis & 8 & $4.57 \times 10^{-2}$ \\
hsa04062 & Chemokine signaling pathway & $4.91 \times 10^{-2}$ \\
\hline
\end{tabular}

KEGG, Kyoto Encyclopedia of Genes and Genomes.

in GO-BP terms associated with cell adhesion and apoptosis (Table V). DEGs in cluster 3 included C5 anaphylatoxin chemotactic receptor 1, GPR18, hydroxycarboxylic acid receptor (HCAR) 2 and HCAR3 (Fig. 2), which were enriched in the G-protein signaling pathway (Table V).

\section{Discussion}

In the present study, a total of 257 DEGs were identified in blood samples from patients with CRPS when compared with healthy controls. Pathway enrichment analysis demonstrated that the identified DEGs were primarily enriched in immune response, cell motion, adhesion and angiogenesis signaling pathways. In the PPI network, EP300 displayed the highest degree and was present in cluster 1. Additional DEGs in cluster 1 with relatively high degrees were STAT3, CREBBP, STAT5A, ITGAM, notch homolog 1, CCAAT/enhancer binding protein $\beta$ and mitogen-activated protein kinase 3 .

Goebel et al (14) demonstrated that intravenous immunoglobulin (IVIG) is effective for the reduction of pain in patients 


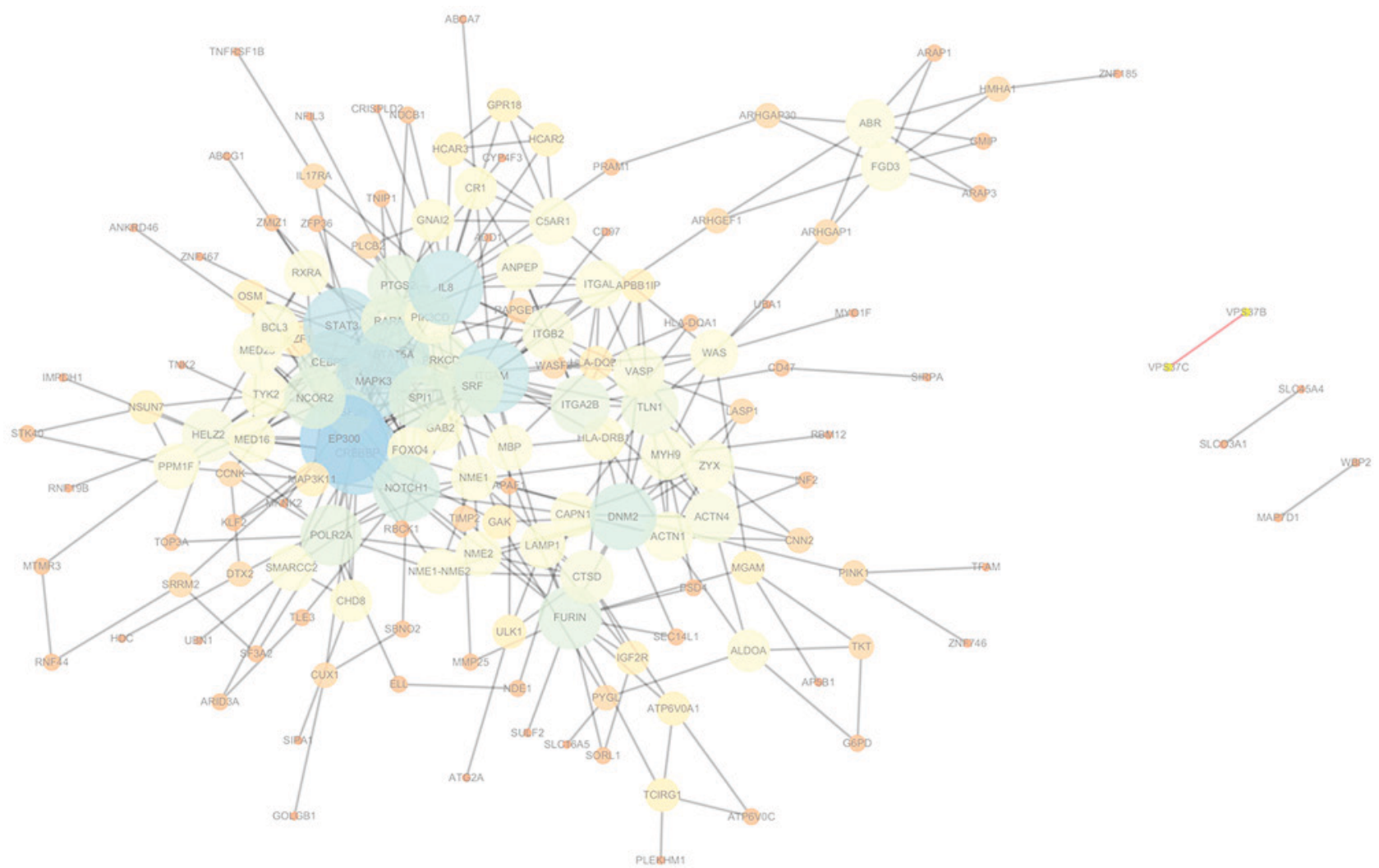

Figure 1. Protein-protein interaction network of differentially expressed genes. Degree is used to describe the number of a node's connection with other nodes. Node size and color represent the degree of proteins. Larger size with a blue color indicates nodes with larger degree, whereas a smaller size with a red color indicates a lower degree.

\section{Cluster 1}

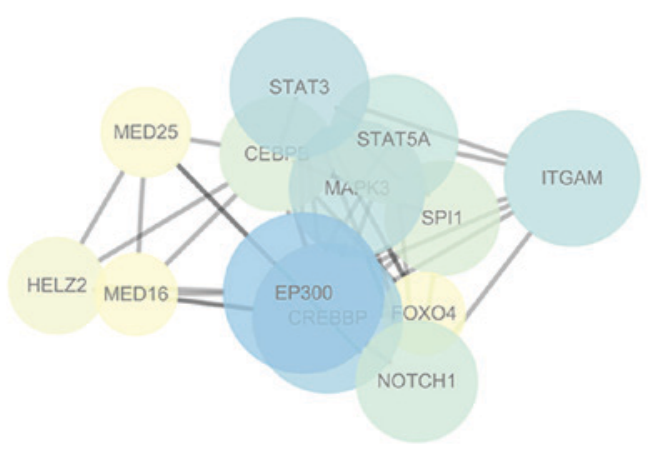

\section{Cluster 2}

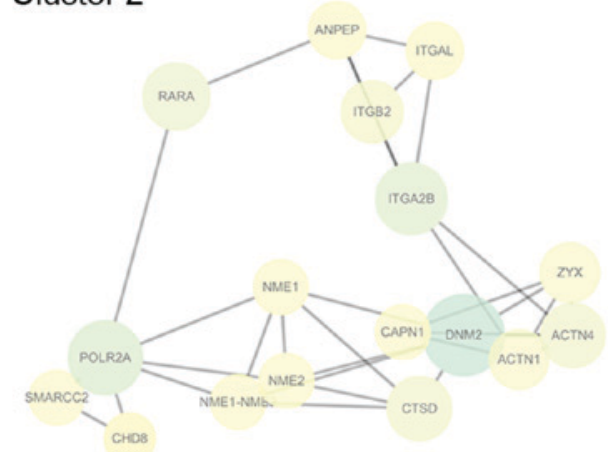

Cluster 3

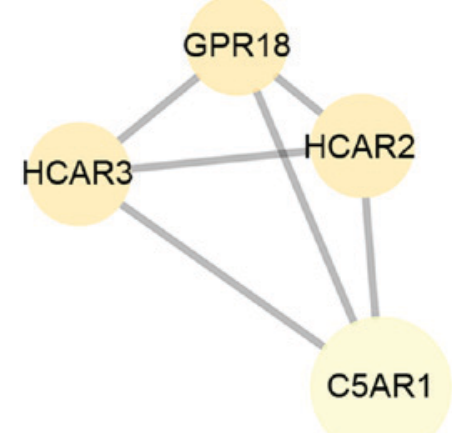

Figure 2. Significant modules in the protein-protein interaction network. Degree is used to describe the number of a node's connection with other nodes. Node size and color represent the degree of proteins. Larger size with a blue color indicates nodes with larger degree, while smaller size with a red color indicates a lower degree. The score calculated by MCODE plugin in the process of module identification to evaluate the size and density of the subgroup for clusters 1,2 and 3 were $8.3,4.3$ and 4.0, respectively. A larger and denser module had a higher score. 
Table V. Significant GO-biological process terms enriched by differentially expressed genes in clusters.

A, Cluster 1

\begin{tabular}{|c|c|c|c|}
\hline GO ID & Biological process & Count & P-value \\
\hline GO:0045941 & Positive regulation of transcription & 8 & $6.02 \times 10^{-8}$ \\
\hline GO:0010628 & Positive regulation of gene expression & 8 & $7.38 \times 10^{-8}$ \\
\hline GO:0045935 & $\begin{array}{l}\text { Positive regulation of nucleobase, nucleoside, } \\
\text { nucleotide and nucleic acid metabolic process }\end{array}$ & 8 & $1.21 \times 10^{-7}$ \\
\hline GO:0051173 & Positive regulation of nitrogen compound metabolic process & 8 & $1.50 \times 10^{-7}$ \\
\hline GO:0010557 & Positive regulation of macromolecule biosynthetic process & 8 & $1.66 \times 10^{-7}$ \\
\hline GO:0031328 & Positive regulation of cellular biosynthetic process & 8 & $2.29 \times 10^{-7}$ \\
\hline GO:0009891 & Positive regulation of biosynthetic process & 8 & $2.52 \times 10^{-7}$ \\
\hline GO:0006357 & Regulation of transcription from RNA polymerase II promoter & 8 & $3.43 \times 10^{-7}$ \\
\hline GO:0045893 & Positive regulation of transcription, DNA-dependent & 7 & $7.40 \times 10^{-7}$ \\
\hline GO:0051254 & Positive regulation of RNA metabolic process & 7 & $7.77 \times 10^{-7}$ \\
\hline GO:0010604 & Positive regulation of macromolecule metabolic process & 8 & $1.05 \times 10^{-6}$ \\
\hline GO:0006350 & Transcription & 10 & $2.11 \times 10^{-6}$ \\
\hline GO:0045944 & $\begin{array}{l}\text { Positive regulation of transcription from RNA } \\
\text { polymerase II promoter }\end{array}$ & 6 & $6.09 \times 10^{-6}$ \\
\hline GO:0006355 & Regulation of transcription, DNA-dependent & 9 & $9.79 \times 10^{-6}$ \\
\hline GO:0051252 & Regulation of RNA metabolic process & 9 & $1.16 \times 10^{-5}$ \\
\hline
\end{tabular}

B, Cluster 2

\begin{tabular}{|c|c|c|c|}
\hline GO ID & Biological process & Count & P-value \\
\hline GO:0007155 & Cell adhesion & 6 & $4.95 \times 10^{-4}$ \\
\hline GO:0022610 & Biological adhesion & 6 & $4.98 \times 10^{-4}$ \\
\hline GO:0007229 & Integrin-mediated signaling pathway & 3 & $2.31 \times 10^{-3}$ \\
\hline GO:0007160 & Cell-matrix adhesion & 3 & $3.70 \times 10^{-3}$ \\
\hline GO:0031589 & Cell-substrate adhesion & 3 & $4.47 \times 10^{-3}$ \\
\hline GO:0042981 & Regulation of apoptosis & 5 & $7.67 \times 10^{-3}$ \\
\hline GO:0043067 & Regulation of programmed cell death & 5 & $7.94 \times 10^{-3}$ \\
\hline GO:0010941 & Regulation of cell death & 5 & $8.05 \times 10^{-3}$ \\
\hline GO:0051017 & Actin filament bundle formation & 2 & $2.15 \times 10^{-2}$ \\
\hline GO:0007159 & Leukocyte adhesion & 2 & $2.86 \times 10^{-2}$ \\
\hline GO:0006338 & Chromatin remodeling & 2 & $5.64 \times 10^{-2}$ \\
\hline GO:0051271 & Negative regulation of cell motion & 2 & $6.33 \times 10^{-2}$ \\
\hline GO:0007015 & Actin filament organization & 2 & $7.20 \times 10^{-2}$ \\
\hline
\end{tabular}

C, Cluster 3

\begin{tabular}{lcrr}
\hline GO ID & Biological process & Count & P-value \\
\hline GO:0007186 & G-protein coupled receptor protein signaling pathway & 2 & $8.30 \times 10^{-2}$ \\
\hline
\end{tabular}

GO, Gene Ontology.

with CRPS, which suggests that the immune response may be important in the development of CRPS. Subsequent studies indicated that neuroautoimmunity and neurogenic inflammation contribute to CRPS. Cooper et al (15) demonstrated that infiltrating leukocytes reacted to autoantibodies that bind to autoantigens located on the surface of neuronal and glial cell targets, and were closely associated with neuroautoimmune responses. In addition, the development of autoantibodies against the $\beta_{2}$ adrenergic receptor and muscarinic- 2 receptor were identified in patients with CRPS (16). 
HLA-DQB1, HLA-DRB4 and HLA-DRB1, which belong to class II of the HLA family, are expressed by a subgroup of immune cells, including B cells, activated T cells and macrophages (17). Kemler et al (18) discovered increased expression of HLA-DQB1 among CRPS patients. In addition, a previous study demonstrated that HLA is associated with CRPS whereby two members of the HLA family (HLA-DQ8 and HLA-B62) were observed to be differentially expressed in different subtypes of CRPS (19). Furthermore, genes in class I of the HLA family were observed to be associated with the development of CRPS (20). HLA-DR2 and HLA-DR13 have been reported to be associated with CRPS $(21,22)$. However, studies in HLA-DRB4 and DRB1 have not focused on their roles in CRPS. Therefore, further research to determine how HLA may be involved in CRPS may provide additional important information. Changes in the expression levels of the HLA family indicates that CRPS may be associated with inflammation regulation (19). Additionally, CRPS may be important to genes closely associated with HLA instead of HLA genes themselves (20).

EP300 and its paralog CREBBP are transcriptional coactivators that regulate gene transcription by connecting DNA-binding and transcription factors, relaxing chromatin through its intrinsic histone acetyltransferase activity and modifying specific transcription factors (23). EP300 and CREBBP have been implicated in a large number of diseases. Lunning et al (24) demonstrated that mutations in EP300 and CREBBP are present in germinal center B-cell lymphomas. Kishimoto et al (25) demonstrated that EP300 and CREBBP may be involved in the pathogenesis of follicular lymphoma. Notably, Seltzer et al (26) indicated that EP300 and CREBBP were associated with postnatal microcephaly. That EP300 and CREBBP have a high degree in the PPI network found by our study may add to the myriad of roles of EP300 and CREBBP in disease.

STAT3 and STAT5 belong to the STAT protein family, which was first discovered as part of the cytokine signaling pathway (27). These proteins demonstrate a significant effect on the immune response and in the process of oncogenesis (27). STAT3A serves multiple roles in cytokine signaling pathways (27). STAT3-knock out mice were incapable of completing gastrulation (28), and conditional STAT3-knock out promotes apoptosis (29). In addition, STAT3 is involved in the differentiation of $\mathrm{CD} 4^{+}$cells to Th17 cells, which protect against invading bacteria and fungi and are involved in the autoimmune response $(30,31)$. Furthermore, STAT3 is a recognized oncogene (32). Similarly, STAT5 serves an important role in the differentiation of $\mathrm{CD}^{+}$cells into Treg cells, which are involved in reducing the immune response and protecting against autoimmune disease $(31,33)$. STAT5 expression deficiency was found to closely associated with tumorigenesis by changing the function of natural killer cells to tumor promotion (34). This implies that STAT may be the potential therapeutic target for CRPS (31).

In conclusion, the results of the present study provided additional evidence in support of the hypothesis that neuroautoimmunity is an important factor for the pathogenesis of CRPS. And significant genes such as HLA-DQB1, HLA-DRB4, HLA-DRB1 from the HLA family, EP300 and its paralog CREBBP, STAT3 and STAT5 from the
STAT family were identified by differentially expressed gene and topological analyses, and module identification of the PPI network constructed from these genes. However, further experiments such as western blotting are required to confirm the changes of expression levels of these genes. Additionally, to fully elucidate the mechanism behind how change in the expression levels of these genes lead to CRPS, further studies are required to determine, which pathways contribute to CRPS. Due to the small sample size of the present study the current findings are limited. Therefore, pooling CRPS samples from different sources in future investigations will increase the sample size and confirm the current findings.

\section{References}

1. Palmer G: Complex regional pain syndrome. Aust Prescr 38: 82-86, 2015.

2. de Mos M, de Bruijn AG, Huygen FJ, Dieleman JP, Stricker BH and Sturkenboom MC: The incidence of complex regional pain syndrome: A population-based study. Pain 129: 12-20, 2007.

3. Stanton-Hicks M, Jänig W, Hassenbusch S, Haddox JD, Boas R and Wilson P: Reflex sympathetic dystrophy: Changing concepts and taxonomy. Pain 63: 127-133, 1995.

4. Bruggeman AW, Oerlemans MH and Frölke JP: Warm and cold complex regional pain syndromes: Differences beyond skin temperature? Neurology 73: 1711-1712, 2009.

5. Bruehl S: Complex regional pain syndrome. BMJ 351: h2730, 2015.

6. Bussa M, Guttilla D, Lucia M, Mascaro A and Rinaldi S: Complex regional pain syndrome type I: A comprehensive review. Acta Anaesthesiol Scand 59: 685-697, 2015.

7. Goebel A and Blaes F: Complex regional pain syndrome, prototype of a novel kind of autoimmune disease. Autoimmun Rev 12: 682-686, 2013.

8. Oaklander AL and Fields HL: Is reflex sympathetic dystrophy/complex regional pain syndrome type I a small-fiber neuropathy? Ann Neurol 65: 629-638, 2009.

9. Barad MJ, Ueno T, Younger J, Chatterjee $\mathrm{N}$ and Mackey S: Complex regional pain syndrome is associated with structural abnormalities in pain-related regions of the human brain. J Pain 15: 197-203, 2014.

10. Jin EH, Zhang E, Ko Y, Sim WS, Moon DE, Yoon KJ, Hong JH and Lee WH: Genome-wide expression profiling of complex regional pain syndrome. PLoS One 8: e79435, 2013.

11. Huang DW, Sherman BT and Lempicki RA: Systematic and integrative analysis of large gene lists using DAVID Bioinformatics resources. Nat Protoc 4: 44-57, 2009.

12. Huang da W, Sherman BT and Lempicki RA: Bioinfromatics enrichment tools: Paths toward the comprehensive functional analysis of large gene lists. Nucleic Acids Res 37: 1-13, 2009.

13. Szklarczyk D, Franceschini A, Wyder S, Forslund K, Heller D, Huerta-Cepas J, Simonovic M, Roth A, Santos A, Tsafou KP, et al: STRING v10: Protein-protein interaction networks, integrated over the tree of life. Nucleic Acids Res 43: D447-D452, 2015.

14. Goebel A: Immunoglobulin responsive chronic pain. J Clin Immunol 30 (Suppl 1): S103-S108, 2010.

15. Cooper MS and Clark VP: Neuroinflammation, neuroautoimmunity, and the co-morbidities of complex regional pain syndrome. J Neuroimmune Pharmacol 8: 452-469, 2013.

16. Kohr D, Singh P, Tschernatsch M, Kaps M, Pouokam E, Diener M, Kummer W, Birklein F, Vincent A, Goebel A, et al: Autoimmunity against the $\beta \backslash 2$ adrenergic receptor and muscarinic-2 receptor in complex regional pain syndrome. Pain 152: 2690-2700, 2011.

17. Klein J and Sato A: The HLA system. First of two parts. N Engl J Med 343: 702-709, 2000.

18. Kemler MA, van de Vusse AC, van den Berg-Loonen EM, Barendse GA, van Kleef M and Weber WE: HLA-DQ1 associated with reflex sympathetic dystrophy. Neurology 53: 1350-1351, 1999.

19. van Rooijen DE, Roelen DL, Verduijn W, Haasnoot GW, Huygen FJ, Perez RS, Perez RS, Claas FH, Marinus J, van Hilten JJ and van den Maagdenberg AM: Genetic HLA associations in complex regional pain syndrome with and without dystonia. J Pain 13: 784-789, 2012. 
20. van de Beek WJ, Roep BO, van der Slik AR, Giphart MJ and van Hilten BJ: Susceptibility loci for complex regional pain syndrome. Pain 103: 93-97, 2003.

21. Mailis A and Wade J: Profile of Caucasian women with possible genetic predisposition to reflex sympathetic dystrophy: A pilot study. Clin J Pain 10: 210-217, 1994.

22. van Hilten JJ, van de Beek WJ and Roep BO: Multifocal or generalized tonic dystonia of complex regional pain syndrome: A distinct clinical entity associated with HLA-DR13. Ann Neurol 48: 113-116, 2000.

23. Wang F, Marshall CB and Ikura M: Transcriptional/epigenetic regulator $\mathrm{CBP} / \mathrm{p} 300$ in tumorigenesis: Structural and functional versatility in target recognition. Cell Mol Life Sci 70: 3989-4008, 2013.

24. Lunning MA and Green MR: Mutation of chromatin modifiers; an emerging hallmark of germinal center B-cell lymphomas. Blood Cancer J 5: e361, 2015.

25. Kishimoto W and Nishikori M: Molecular pathogenesis of follicular lymphoma. J Clin Exp Hematop 54: 23-30, 2014.

26. Seltzer LE and Paciorkowski AR: Genetic disorders associated with postnatal microcephaly. Am J Med Genet C Semin Med Genet 166C: 140-155, 2014.

27. Lim CP and Cao X: Structure, function, and regulation of STAT proteins. Mol Biosyst 2: 536-550, 2006.

28. Takeda K, Noguchi K, Shi W, Tanaka T, Matsumoto M, Yoshida N, Kishimoto T and Akira S: Targeted disruption of the mouse Stat 3 gene leads to early embryonic lethality. Proc Natl Acad Sci USA 94: 3801-3804, 1997.
29. Takeda K, Kaisho T, Yoshida N, Takeda J, Kishimoto T and Akira S: Stat3 activation is responsible for IL-6-dependent T cell proliferation through preventing apoptosis: Generation and characterization of $\mathrm{T}$ cell-specific Stat3-deficient mice. J Immunol 161: 4652-4660, 1998.

30. Laurence A and O'Shea JJ: T(H)-17 differentiation: Of mice and men. Nat Immunol 8: 903-905, 2007.

31. Ross JA, Nagy ZS, Cheng H, Stepkowski SM and Kirken RA: Regulation of T cell homeostasis by JAKs and STATs. Arch Immunol Ther Exp (Warsz) 55: 231-245, 2007.

32. Bromberg JF, Wrzeszczynska MH, Devgan G, Zhao Y, Pestell RG, Albanese C and Darnell JE Jr: Stat3 as an oncogene. Cell 98: 295-303, 1999.

33. Yao Z, Kanno Y, Kerenyi M, Stephens G, Durant L, Watford WT, Laurence A, Robinson GW, Shevach EM, Moriggl R, et al: Nonredundant roles for $S t a t 5 a / b$ in directly regulating Foxp3. Blood 109: 4368-4375, 2007.

34. Gotthardt D, Putz EM, Grundschober E, Prchal-Murphy M, Straka E, Kudweis P, Heller G, Bago-Horvath Z, Witalisz-Siepracka A, Cumaraswamy AA, et al: STAT5 is a key regulator in NK cells and acts as a molecular switch from tumor surveillance to tumor promotion. Cancer Discov 6: 414-429, 2016. 